\title{
POLARIZATION CONVERSION INDUCED IN A NON-CONVENTIONALLY BIASED CENTROSYMMETRIC PHOTOREFRACTIVE CRYSTAL
}

\author{
Claudio Crognale, Luigi Rosa \\ CNX S.P.A. Siemens, S.S.17, Località Boschetto, 67100, L'Aquila, Italy \\ claudio.crognale@siemens.com \\ luigi.rosa@siemens.com
}

Abstract: In an non-conventionally biased $\mathrm{m} 3 \mathrm{~m}$ centrosymmetric photorefractive
crystal, the combination of the internal photorefractive field with the external
static electric field can introduce a conversion between the transverse vector
components of the read-out beam at infrared wavelengths.

In the last years, a great interest has been devoted to the photorefractive materials, with a particular attention to the class of $\mathrm{m} 3 \mathrm{~m}$ centrosymmetric crystals [1]. Recent works carried on biased Potassium Lithium Tantalate Niobate (KLTN) crystals in paraelectric phase have shown that, in these crystals, after the waveguide formation at the visible wavelengths, the internal charge field created by means of the photorefractively active light is still present even when the crystal is illuminated with an infrared beam. Then the quadratic response of the material nonlinearly combines the internal photorefractive field with any external static electric field, so that the index pattern generated by the active light can be strongly modified, up to produce an antiguiding effect on an infrared probe beam [2] (i.e. the read-out field). It is our opinion that even more attractive properties of such photorefractives could be highlighted, when non-conventional biasing configurations are taken into account. In this work, we introduce a non-conventional scheme, which allows to vary the orientation of the external biasing static field respect to that of the optical field vector, thus enhancing the tensorial features of the crystal. We found that, by imposing a proper non-conventional boundary to the crystal, after the waveguide formation, the combination of the internal photorefractive field with the external static electric field can properly modify the bulk refractive index pattern, introducing an attractive conversion mechanism between the transverse vector components of the probe beam at longer wavelengths. 


\subsection{THEORETICAL MODEL.}

Let us consider a visible optical beam polarized in the $x-y$ plane that propagates along the $z$ axis of a centrosymmetric photorefractive crystal [3]:

$$
\vec{A}(x, y, z)=\hat{x} A_{X}(x, y, z) e^{i k_{X} z-i \omega t}+\hat{y} A_{Y}(x, y, z) e^{i k_{Y} z-i \omega t}+c . c .
$$

with a Gaussian input field spatial distributions $A_{X, Y}(x, y, 0)$ [2]. We assume that the $x-y$ transverse polarization plane and the propagation direction of the optical field are according to an $(\mathrm{x}, \mathrm{y}, \mathrm{z})$ coordinates system oriented along the principal dielectric axes of the crystalline medium as well. Moreover, we consider a centrosymmetric biased by means of a purely transverse (i.e. independent on the $z$ axis) external static field distribution. At the steady-state, the propagation of the transverse Gaussian needle beam through the biased photorefractive can be described by means of the Kukhtarev's and Helmoltz's nonlinear coupled equations [3], [4], [5] (by assuming the validity of the slowly varying approximation for the field amplitudes $A_{X, Y}$ ):

$$
\begin{aligned}
& \nabla \cdot\left[\left(I_{0}+I_{B}\right) \nabla \vartheta_{0}-\frac{K_{B} T}{q} \nabla\left(I_{0}+I_{B}\right)\right]=0 \\
& \frac{\partial A_{X}}{\partial z}-\frac{i}{2 k_{X}}\left(\frac{\partial^{2} A_{X}}{\partial x^{2}}+\frac{\partial^{2} A_{X}}{\partial y^{2}}\right)=\frac{i k_{X}}{n_{X}} \Delta n_{11} A_{X}+\frac{i k_{X}}{n_{X}} \Delta n_{12} A_{Y} \exp \left[i\left(k_{X}-k_{Y}\right) z\right] \\
& \frac{\partial A_{Y}}{\partial z}-\frac{i}{2 k_{Y}}\left(\frac{\partial^{2} A_{Y}}{\partial x^{2}}+\frac{\partial^{2} A}{\partial y^{2}}\right)=\frac{i k_{y}}{n_{y}} \Delta n_{21} A_{X} \exp \left[-i\left(k_{Y}-k_{Y}\right) z\right]+\frac{i k_{Y}}{n_{Y}} \Delta n_{22} A_{Y},
\end{aligned}
$$

In the expressions above, $\nabla$ is the transverse gradient operator, $\vartheta_{0}$ is the local electrostatic potential (according to the assigned boundary conditions), $I_{0}(x, y, z)=|A(x, y, z)|^{2}=\left|A_{X}(x, y, z)\right|^{2}+\left|A_{Y}(x, y, z)\right|^{2}$ the optical intensity of the propagating beam in nonlinear regime, $I_{B}$ the artificial dark irradiance (constant), $K_{B}$ the Boltzmann constant, $T$ the absolute temperature, $q$ the electron charge, $n_{x}$ and $n_{y}$ the zero-field principal refractive indices for light polarized along the $x$ and $y$ principal dielectric axes of the crystal, respectively, $k_{x}=k n_{x}$, $k_{y}=k n_{y}$, in which $k=\frac{2 \pi}{\lambda}$ is the vacuum wave number ( $\lambda$ is the vacuum wavelength). We take in account the tensorial nature of the crystal by means of the terms $\Delta n_{i j}$ in (3a), (3b), which are associated to the refractive index modulation due to the local space-charge field distribution, induced by the photorefractive effect. Without any lack of generality, let us consider for our investigation an $\mathrm{m} 3 \mathrm{~m}$ 
cubic centrosymmetric structure, such as the Potassium LithiumTantanate Niobate (KLTN, [2]). Thanks to its symmetry properties, in such a medium $n_{x}=n_{y}$, and the tensorial properties are completely described by one value of the relative dielectric constant $\varepsilon_{r}$, and by only three values of the components of the electrooptic quadratic tensor: $g_{11}=g_{22}, g_{12}=g_{21}$, and $g_{66}$, because the other terms $g_{16}=g_{61}=g_{62}=g_{26}=0$. The nonlinear terms in (3a), (3b) can be written as [6]:

$$
\begin{aligned}
& \Delta n_{11}=-\frac{1}{2} n_{x}^{3} \varepsilon_{0}^{2}\left(\varepsilon_{r}-1\right)^{2}\left(g_{11} E_{x}^{2}+2 g_{16} E_{x} E_{y}+g_{12} E_{y}^{2}\right) \\
& \Delta n_{12}=-\frac{1}{2} n_{x}^{2} n_{y} \varepsilon_{0}^{2}\left(\varepsilon_{r}-1\right)^{2}\left(g_{61} E_{x}^{2}+2 g_{66} E_{x} E_{y}+g_{62} E_{y}^{2}\right) \\
& \Delta n_{21}=-\frac{1}{2} n_{x} n_{y}^{2} \varepsilon_{0}^{2}\left(\varepsilon_{r}-1\right)^{2}\left(g_{61} E_{x}^{2}+2 g_{66} E_{x} E_{y}+g_{62} E_{y}^{2}\right) \\
& \Delta n_{22}=-\frac{1}{2} n_{y}^{3} \varepsilon_{0}^{2}\left(\varepsilon_{r}-1\right)^{2}\left(g_{21} E_{x}^{2}+2 g_{26} E_{x} E_{y}+g_{22} E_{y}^{2}\right)
\end{aligned}
$$

In (4a)-(4d), $\varepsilon_{0}$ is the absolute dielectric constant, $E_{x}$ and $E_{y}$ the component of the local static space-charge electric field in the biased medium. We have performed our analysis by numerically solving the nonlinear propagation in (3a), (3b) (4a)-(4d) with the Beam Propagation Method (BPM), and the potential equation in (2) with a 5 points Finite Difference Method (FDM). The boundary conditions for the potential $\vartheta_{0}$ were assigned by means of the external biasing voltage $V_{B}(x, y)$ applied to the crystal. For our analysis, we have introduced a particular theoretical model in which we assigned, in correspondence of the transverse section perpendicular to the $\mathrm{z}$ axis, the following boundary conditions for the potential $\vartheta_{0}$ :

$$
\begin{array}{ll}
V_{B}\left(x=-\frac{L_{X}}{2}, y\right)=\left(\frac{V_{2}}{L_{Y}}\right)\left(y+\frac{L_{Y}}{2}\right)+V_{B 0} & -\frac{L_{Y}}{2} \leq y \leq \frac{L_{Y}}{2} \\
V_{B}\left(x=\frac{L_{X}}{2}, y\right)=\left(\frac{V_{2}}{L_{Y}}\right)\left(y+\frac{L_{Y}}{2}\right)+V_{B 0}+V_{1} & -\frac{L_{Y}}{2} \leq y \leq \frac{L_{Y}}{2} \\
V_{B}\left(x, y=+\frac{L_{Y}}{2}\right)=\left(\frac{V_{1}}{L_{X}}\right)\left(x+\frac{L_{X}}{2}\right)+V_{B 0}+V_{2} & -\frac{L_{X}}{2} \leq x \leq \frac{L_{X}}{2} \\
V_{B}\left(x, y=-\frac{L_{Y}}{2}\right)=\left(\frac{V_{1}}{L_{X}}\right)\left(x+\frac{L_{X}}{2}\right)+V_{B 0} & -\frac{L_{X}}{2} \leq x \leq \frac{L_{X}}{2}
\end{array}
$$

In (5a)-(5d), $L_{X}$ and $L_{Y}$ are the transversal sizes of the boundary, and $V_{B 0}, V_{1}$, 
$V_{2}$ the boundary voltage values that are assumed independent on the $\mathrm{x}$ and $\mathrm{y}$ coordinates. From a practical point of view, this approach is equivalent to consider a crystal with transverse sizes much more larger than $L_{X}$ and $L_{Y}$, that is biased by means of two independent, perpendicularly oriented external static fields, respectively given by $\vec{E}_{X b}=-\hat{x} \frac{V_{1}}{L_{X}}, \vec{E}_{Y b}=-\hat{y} \frac{V_{2}}{L_{Y}}$, when the beam illumination is absent. Moreover, we also assume in this model that the transversal sizes of the boundary are much larger than the beam spot diameter, so that the presence of the optical beam field does not change the distribution of the electric static field at the boundary. According to this approach, we considered transverse boundary sizes $L_{x}, L_{y}$ at least $20 \times(\Delta x, \Delta y)(\Delta x, \Delta y$ are the Full Widths at Half Maximum of the optical field intensity along $\mathrm{x}$ and $\mathrm{y}$ directions, respectively).

\subsection{NUMERICAL RESULTS AND DISCUSSION}

Now consider a Gaussian read-out probe field at the input of the crystal after the waveguide associated to $\Delta n_{i j}(x, y)$ is formed. According to [2], in the numerical simulation, we study the propagation of a probe beam with the same wavelength of the active beam. This approach does not introduce any lack of generality, and all results reported below can be extended to other investigations in which longer wavelength optical probe beams are considered. The propagation of the read-out field through the biased photorefractive can be described by numerically solving the corresponding set of coupled equations (3a), (3b), with the (4a)-(4d), in which the effective read-out static electric field components are inserted:

$$
\begin{aligned}
& E_{x e f f}=E_{x}-E_{x b}+E_{x m}, \\
& E_{y e f f}=E_{y}-E_{y b}+E_{y m},
\end{aligned}
$$

In (6a), $(6 \mathrm{~b}), E_{x m}, E_{y m}$ are the external biasing read-out static electric field vector components. We considered a $4 \mathrm{~mm}$-long KLTN crystal, according to the conditions (reported in [2]): $\Delta x=\Delta y=10 \mu m$ for both spatial distributions $A_{X, Y}(x, y, 0), \quad I_{P X} / I_{B}=I_{P Y} / I_{B}=1.3 \quad\left(I_{P X}, \quad I_{P Y} \quad\right.$ are the peak intensities of the orthogonal polarization states), $\lambda=532 \mathrm{~nm}, n_{x}=n_{y}=2.4$, $\mathcal{E}_{r}(293 K)=9000, \quad g_{11}=g_{22}=0.12 m^{4} C^{-2}, \quad g_{12}=g_{21}=0.02 m^{4} C^{-2}$, $g_{66}=0.05 m^{4} C^{-2}$. The value of term $g_{66}$ for the KLTN is not available from the 
literature. Then, in our theoretical model, we have assumed this component to be of the order of the term $g_{66}$ of the Barium Titanate $\left(\mathrm{BaTiO}_{3}\right)$ electro-optic quadratic tensor, because this crystal exhibits in the paraelectric phase the same structure of the KLTN [6]. The crystal was biased by means of two independent, perpendicularly oriented external static fields with the same magnitudes, equal to $E_{x b}=E_{y b}=3 \cdot 10^{5} \mathrm{~V} / \mathrm{m}$. In the read-out phase, we imposed $E_{x m}=E_{x b}=3 \cdot 10^{5} \mathrm{~V} / \mathrm{m}, \quad E_{y m}=E_{y b}=3 \cdot 10^{5} \mathrm{~V} / \mathrm{m}, \quad$ and $I_{P O X} / I_{B}=2, I_{P O Y} / I_{B}=0\left(I_{P O X}, I_{P 0 Y}\right.$ are the peak intensities of the probe field orthogonal vector components). In this way, we simulated the propagation of a read-out Gaussian beam with the optical electric field vector lying just along the $\mathrm{x}$ axis. To analyze the result of our theoretical study, let us introduce the following parameters $P_{0 X}(z), P_{0 Y}(z)$ :

$P_{0 X, 0 Y}(z)=\frac{\iint I_{0 X, 0 Y}(x, y, z) d x d y}{\iint\left(I_{0 X}(x, y, 0)+I_{0 Y}(x, y, 0)\right) d x d y}$

$P_{0 X}(z), P_{0 Y}(z)$ are, respectively, the percentages of optical power contained in the $\mathrm{x}$ and $\mathrm{y}$ read-out optical field vector components at the crystal length $\mathrm{z}$. The result is summarized in Fig.1.

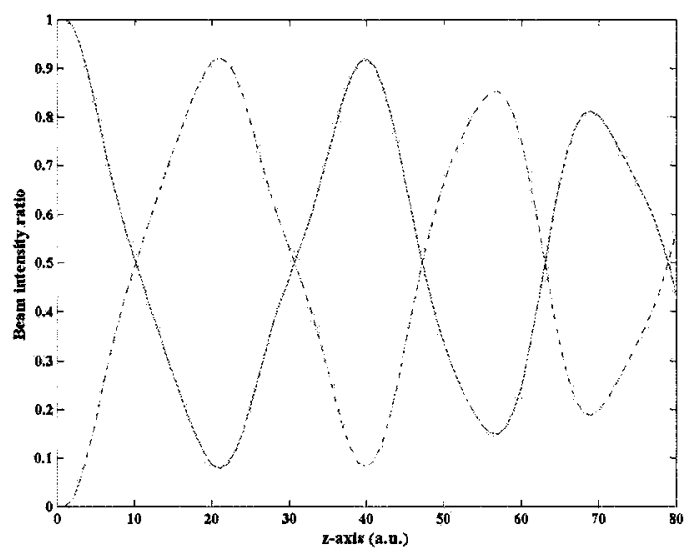

Figure 1. Power exchange between polarization vector components

$$
\left(E_{x m}=E_{x b}=3 \cdot 10^{5} \mathrm{~V} / \mathrm{m}, E_{y m}=E_{y b}=3 \cdot 10^{5} \mathrm{~V} / \mathrm{m}\right)
$$

This figure reports the values of $P_{0 X}(z)$ (continuous curve), $P_{0 Y}(z)$ (dashed- 
dotted curve) obtained according to the read-out launching conditions expressed above (in all figures reported here, a.u. stands for arbitrary units). It clearly appears a quasi-periodic exchange of power between the transverse vector components, with a quite good efficiency (higher than $90 \%$ ) of the conversion process for $z=21$ a.u. (corresponding to around $1 \mathrm{~mm}$ propagation distance). Figs. 2(a), 2(b) show the spatial intensity distributions of both orthogonal polarization vectors at this distance (x-polarized component in Figs. 2(a), ypolarized component in Fig. 2(b)).
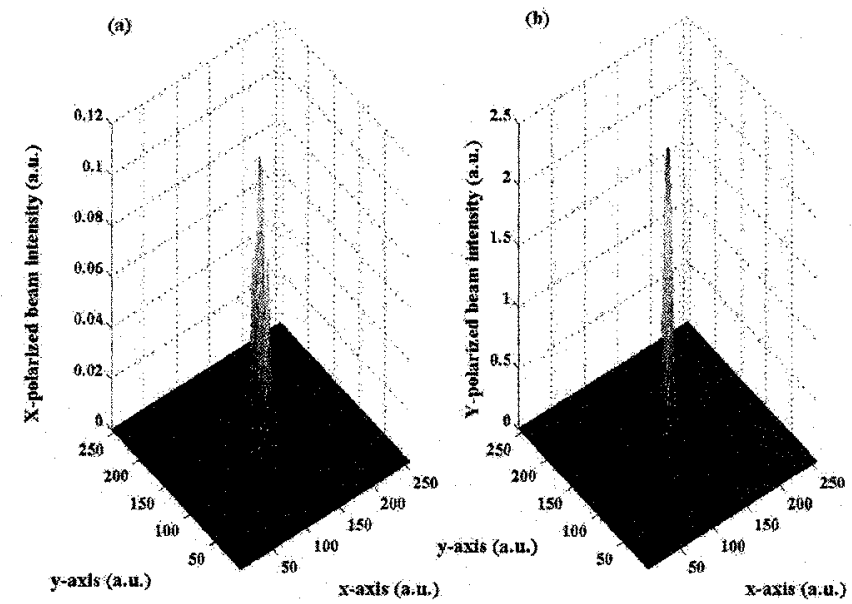

Figure 2. Spatial intensity distributions of orthogonal polarization vectors at $z=21 a . u .\left(E_{x m}=E_{x b}=3 \cdot 10^{5} \mathrm{~V} / \mathrm{m}, E_{y m}=E_{y b}=3 \cdot 10^{5} \mathrm{~V} / \mathrm{m}\right)$ 


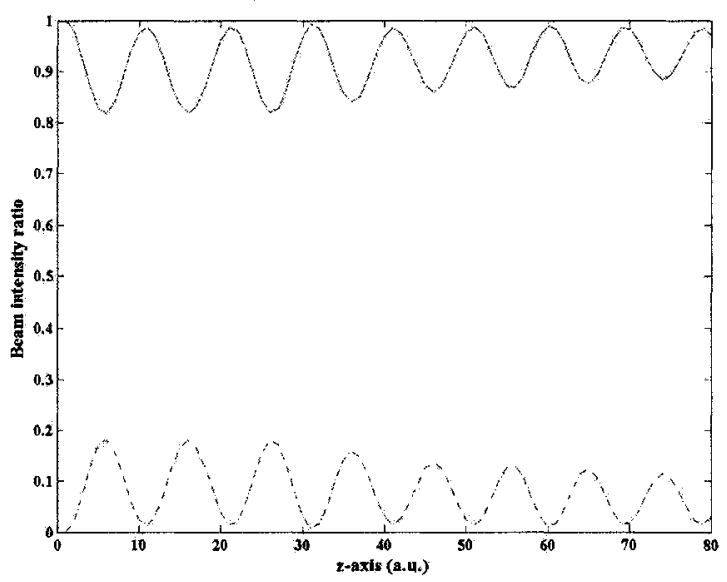

Figure 3. Power exchange between polarization vector components

$$
\left(E_{x b}=E_{y b}=3 \cdot 10^{5} \mathrm{~V} / \mathrm{m}, E_{x m}=0, E_{y m}=0\right)
$$

The impact of the diffraction is quite low, at this distance, and the optical beam spot of the y-polarized component results well focused on the crystal transverse section.

Now let us consider the result in Fig. 3 (the continuous curve is referred to $P_{0 x}(z)$, the dashed-dotted curve to $\left.P_{0 Y}(z)\right)$. We obtained this behavior by launching the $\mathrm{x}$ polarized optical Gaussian probe beam at the input of the unbiased crystal ( $E_{x b}=E_{y b}=3 \cdot 10^{5} \mathrm{~V} / \mathrm{m}, E_{x m}=E_{y m}=0$ ). This figure shows that, in absence of an external biasing field, a quasi-periodic mutual energy exchange between the orthogonal vector components of the linearly polarized read-out field occurs. Nevertheless, at the distance of $z=21$ a.u.considered above, the conversion process is extremely low (less than $5 \%$ ) so that, in this case, the $x$ polarized component appears well focused on the crystal transverse section. This is shown in Figs. 4(a), 4(b), where the spatial intensity distributions of both orthogonal polarization vectors at this distance are reported (x-polarized component in Fig.4(a), y-polarized component in Fig. 4(b)). 

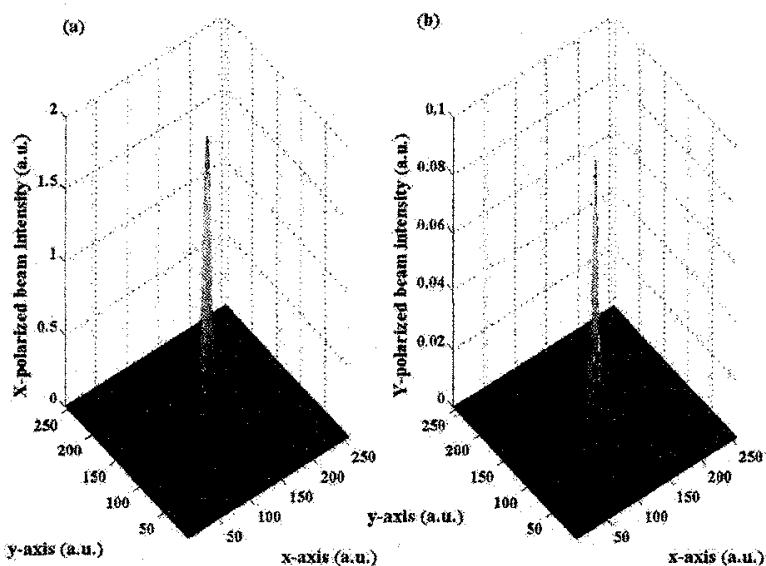

Figure 4. Spatial intensity distributions of orthogonal polarization vectors at $z=21 a . u .\left(E_{x b}=E_{y b}=3 \cdot 10^{5} \mathrm{~V} / \mathrm{m}, E_{x m}=0, E_{y m}=0\right)$

\section{REFERENCES}

[1] G. M. Tosi-Beleffi, F. Curti, D. Boschi, C. Palma and A. J. Agranat, "Soliton-based Ybranch in photorefractive crystals induced through dispersion-shifted optical fiber," Opt. Lett., vol. 28, pp. 1561-1563, 2003

[2] E. DelRe, M. Tamburrini and A. J. Agranat, "Soliton electro-optic effects in paraelectrics," Opt. Lett., vol. 25, pp. 963-965, 2000

[3] M.Segev, G. C. Valley, S. R. Singh, M. I. Carvalho, andD. N. Christodoulides, "Vector photorefractive spatial solitons" Opt. Lett., vol. 20, pp. 1764-1766, 1995

[4] B. Crosignani, P. Di Porto, A. Degasperis, M. Segev, and S. Trillo, "Three-dimensional optical beam propagation and solitons in photorefractive crystals," J. Opt. Soc. Am. B, vol. 14, pp. 3078-3090, 1997

[5] B. Crosignani, P. Di Porto, M. Segev, G. Salamo, and A. Yariv, Rivista del Nuovo Cimento, vol. 21, pp.1-37, 1998

[6] A. Yariv and P. Yeh, Optical Waves in Crystals: Propagation \& Control of Laser Radiation, Ed. New York: John Wiley \& Sons, 1983 